\title{
Water Outburst Activity in Comet 17P/Holmes
}

\author{
Amaury A. de Almeida ${ }^{a}$, Daniel C. Boice ${ }^{a, b, c,}{ }^{*}$, Enos Picazzio ${ }^{a}$, Walter F. Huebner ${ }^{d}$
}

\begin{abstract}
Cometary outbursts are sporadic events whose mechanisms are not well known where the activity and consequently the brightness can increase hundreds of thousands of times within a few hours to several days. This indicates a dramatic departure from thermal equilibrium between the comet and interplanetary space and is usually documented by "light curves". In a typical cometary outburst, the brightness can increase by 2-5 magnitudes (Whitney, 1955; Gronkowski and Wesolowski, 2015). In only 42 hours, Comet $17 \mathrm{P} /$ Holmes was reported to brighten from a magnitude of about 17 to about 2.4 at the height of the burst, representing the largest known outburst by a comet. We present the $\mathrm{H}_{2} \mathrm{O}$ production rate of Holmes for the megaburst occurring between 23 and 24 October 2007. For this, we selected more than 1900 photometric observations from the International Comet Quarterly Archive of Photometric Data and use the Semi-Empirical Method of Visual Magnitudes (SEMVM; de Almeida et al., 2007). We clearly show that the comet achieved an average water production rate of $5 \times 10^{29}$ molecules $\mathrm{s}^{-1}$, corresponding to a water gas loss rate of $14,960 \mathrm{~kg} \mathrm{~s}^{-1}$, in very good agreement with Schleicher (2009) who derived the water production rate using $\mathrm{OH}$ measurements on 1 Nov 2007 (about 8 days after the outburst). We discuss possible physical processes that might cause cometary outbursts and propose a new qualitative mechanism, the Pressurized Obstructed Pore (POP) model. The key feature of $\mathrm{POP}$ is the recrystallization of water in the surface regolith as it cools, plugging
\end{abstract}


pores and blocking the release of subsurface gas flow. As the interior gas pressure increases, an outburst is eventually triggered. POP is consistent with current observations and can be tested in the future with observations (e.g., Rosetta in situ measurements) and detailed simulations.

Keywords: Comet 17P/Holmes; Jupiter-family comets; water release rates; cometary outbursts

\section{Introduction}

The correlation between visual magnitudes and water production rates, $\mathrm{Q}\left(\mathrm{H}_{2} \mathrm{O}\right)$, is useful for studying the activity of comets from data sets of visual magnitudes. In the present work, we use the visual magnitudes from the International Comet Quarterly (ICQ) Archive of Photometric Data to derive $\mathrm{Q}\left(\mathrm{H}_{2} \mathrm{O}\right)$ for the 2007 outburst of 17P/Holmes.

The Jupiter-family comet (JFC), 17P/Holmes (orbital period of about 6.89 years), underwent a major outburst on 23.8 October 2007 UT, 5 months after perihelion (at 2.05 AU on 4.5 May 2007 UT), reaching maximum brightness (from $m_{v}=16.5$ to $m_{v}=2.5$ ) about 1.7 days (42 hours) after the event and becoming a naked-eye object for several months. Holmes had exhibited earlier outbursts on 6 November 1892 and 16 January 1893. In total, this increase in brightness of 14 magnitudes, the largest known outburst of a comet so far, occurred 173 days after perihelion passage, at a distance of $2.44 \mathrm{AU}$ from the Sun and $1.63 \mathrm{AU}$ from the Earth.

The most favorable scenario for the outburst is that the comet was heated by solar radiation during its perihelion passage, and deep layers of icy volatiles were sublimated by the inward propagation of the thermal wave. Later, the internal gas pressure produced by sublimation disrupted the comet surface, leading to a dramatic emission of gas and dust causing the outburst of the comet. Here we study the activity of 17P/Holmes before and after the 2007 
outburst through optical magnitude observational data, since the majority of comet outbursts are detected and recorded visually.

\section{Molecular production rates}

Production of gas-phase molecules is largely responsible for cometary activity. Near the Sun, it is mainly the cometary wind of $\mathrm{H}_{2} \mathrm{O}$ molecules that lifts the dust particles from the nucleus, so a correlation between $\mathrm{H}_{2} \mathrm{O}$ and dust production is expected (Altenhoff et al., 2009; Sanzovo et al., 2010).

If $m^{\prime}{ }_{v}\left(=m_{6.78}-5 \log \Delta\right)$ is the total visual magnitude observed from the coma of a comet, reduced to the standard aperture diameter of $6.78 \mathrm{~cm}$ when the observer is placed at a standard geocentric distance $\Delta=1 \mathrm{AU}$ (Morris, 1973) and the comet at a heliocentric distance $r$ (in AU and less than about $3 \mathrm{AU}$ ), the water production rate (in molecules $\mathrm{s}^{-1}$ ) is given by (Newburn, 1981; de Almeida et al., 1997; Sanzovo et al., 2001)

$$
Q\left(H_{2} O\right)=\left\{\frac{r^{2} \cdot 10^{\left[0.4\left(-26.8-m_{v}^{\prime}\right)\right]}-p \cdot R_{N}^{2} \phi_{N}}{l_{r} \cdot R[1+\delta(r, \theta)]}\right\}^{0.825}
$$

where $p=p(\lambda)$ is the geometric visual albedo of the nucleus with radius $R_{N}$, and $\phi_{N}=0.9982 \mathrm{e}^{-1.842 \alpha}$, where $\alpha$ is the phase angle (in rad) of the comet (Sanzovo et al., 1996; Singh et al., 1997), and $I_{r}\left(=6.6 \times 10^{4} \cdot r^{2}\right)$ is the scale-length (in $\mathrm{km}$ ) for photodissociation of the $C_{2}$ radical (Randall et al., 1992; A'Hearn et al., 1995). $A^{\prime}$ Hearn et al. (1995) showed that JFCs are carbon-depleted having low abundances of carbon-chain species like $\mathrm{C}_{2}, \mathrm{C}_{3}$, and $\mathrm{CN}$ when compared to Halley-type comets (HTC) and long-period comets (LPC). In Eq. (1), the exponent $(0.825)$ represents an average correlation between water release rates and $\mathrm{C}_{2}$ production rates, obtained using observational data from Comet Levy (C/1990 K1) and seven JFCs (6P/d'Arrest, 9P/Tempel 1, 10P/Tempel 2, 22P/Kopff, 26P/Grigg-Skjellerup, 67P/Churyumov-Gerasimenko, and 81P/Wild 2), as reported by Sanzovo et al. (2001) and de Almeida et al. (2007). The parameter $R$ is the resonance fluorescence efficiency that takes into account the water release rate, for an assumed value of the radius $R_{N}$, and is determined empirically, varying between $\sim 10^{-39}$ and $\sim 10^{-37} \mathrm{~cm}^{2} \cdot \mathrm{s}$ according to the periodicity of the comet (de Almeida et al., 1997). If we know the 
fluorescence processes by which $C_{2}$ Swan bands are formed (see, e.g., Rousselot et al., 2000, and other references therein), we might be able to derive a theoretical value for $R$. In Eq. (1), the nucleus only makes a significant contribution when the phase angle $\alpha$ of the comet is small. For the 2007 return of Comet 17P/Holmes, ICQ observational data provide phase angles varying between $\sim 11^{\circ}$ and $\sim 27^{\circ}$. Therefore, the nuclear contribution $\left(\phi_{N}\right)$ that appears in Eq. (1) was considered and the value $R=1.698 \times 10^{-38} \mathrm{~cm}^{2} \cdot \mathrm{s}$ was adopted, following the prescription of de Almeida et al. (1997). The dust-to-gas ratio in a comet can be expressed by (Ney, 1982) as

$$
\delta(r, \theta)=\frac{\delta(r, 90) \delta(\theta)}{\delta(90)}
$$

in which the scattering function $\delta(\theta)$ is obtained from the observational curve proposed by Divine (1981), for the scattering angle $\theta=180^{\circ}-\alpha$, and $\delta(90)=$ 0.0362 . For detailed descriptions of the functions, $\delta(r, 90)$ and $\delta(\theta)$ in Eq. 1 , see Devine (1981), Ney (1982), and de Almeida et al. (1997). We have determined and applied in Eq. (1) the dependences of $\delta(r, 90)$ with $r$ for each individual comet studied by Sanzovo et al. (2001) and de Almeida et al. (2007), and included observational data from Schleicher (2009) for the 2007 return of Comet 17P/Holmes. This is the basis of our Semi-Empirical Method of Visual Magnitudes (SEMVM, de Almeida et al., 2007) model. Our derived water production rates are given in Figure 1 before and after the outburst and selected values are given in Table 1 with comparisons to the observational conditions of Schleicher (2009).

(insert Figure 1)

(insert Table 1)

We note that the hydroxyl radical $(\mathrm{OH})$ production rates (in molecules $\mathrm{s}^{-1}$ ) represent about $85 \%$ of the photodissociation products of water (Huebner, Keady \& Lyon, 1992), so the water production rates are obtained using the relation

$$
Q\left(\mathrm{H}_{2} \mathrm{O}\right)=\frac{Q(\mathrm{OH})}{0.85} .
$$


This allows us to compare with Schleicher (2009) who observed $\mathrm{OH}$ in Holmes $5 \times 10^{29}$ molecules s$^{-1}$, consistent with our results (see Table 1 ).

Once the water production rates are obtained, the conversion to total gas mass loss rates can be made, considering a typical gaseous mixture of $\sim 77 \% \mathrm{H}_{2} \mathrm{O}, \sim 13 \% \mathrm{CO}$, and $\sim 10 \%$ of other molecular species with average molecular weight $\sim 30$ amu (Sanzovo et al., 1996; Singh et al., 1997). Following this, the total gas loss rates (in $\mathrm{kg} \mathrm{s}^{-1}$ ) correlate to the water production rates (in molecules $\mathrm{s}^{-1}$ ) through the expression (Sanzovo et al., 1996)

$$
q_{g}=3.41 \times 10^{-26} \cdot Q\left(\mathrm{H}_{2} \mathrm{O}\right) \text {. }
$$

The total dust production rate can be estimated using the method of Sanzovo et al. (2010). They found a strong correlation between gas and dust production rates for 12 Jupiter-family comets and another one to two longperiod comets, and $1 \mathrm{P} /$ Halley due to its productivity. We applied this later relationship $\left\{\log \left(q_{d}\right)=\left[\log \left(q_{g}\right)+0.200\right] / 1.067\right\}$ for $17 \mathrm{P} /$ Holmes to estimate its dust mass loss rate and dust-to-gas ratio. Selected values of gas and dust production rate estimates are given in Table 2, along with the total mass loss rates (gas and dust) of ejected materials and the dust-to-gas mass ratio $(\chi)$ prior to and following the megaburst.

(insert Table 2)

Besides the water (gas) and dust production rates, SEMVM can be combined with water sublimation theory, described by Cowan and A'Hearn (1979) and Delsemme (1982), in order to obtain an estimate of the minimum and effective nuclear dimension of a comet. If $A_{A}$ is the total active surface area on the sunlit side of the nucleus at a heliocentric distance $r$, and $Z(T)$ is the specific sublimation rate of this volatile (molecules $\mathrm{s}^{-1} \mathrm{~cm}^{-2}$ ) then $A_{A}=f_{A A} \cdot A_{T o t}=$ $Q\left(\mathrm{H}_{2} \mathrm{O}\right) / Z(T)$, where $Q\left(\mathrm{H}_{2} \mathrm{O}\right)$ is the water production rate (in molecules $\mathrm{s}^{-1}$ ) inferred through SEMVM for that same heliocentric distance, and $f_{A A}$ is the fraction of active surface area. Assuming a slow rotation hypothesis for the nucleus with spherical symmetry $\left(A_{\text {Tot }}=2 \pi R_{N}^{2}\right)$, the active surface area on the sunlit side will be bound to the comet's minimum radius by (de Almeida et al., 1997) 


$$
\mathrm{f}_{\mathrm{AA}}=\frac{A_{A}}{2 \pi\left(R_{N}\right)^{2}} \leq 1
$$

Note that $f_{A A}$ can exceed 1 (up to a maximum of 2 ) if there is significant gas emission from the night side since we assume that gas production comes from the sunlit hemisphere. Once the $f_{A A}$ parameter is fixed, sublimation theory (Cowan and A'Hearn, 1979; Delsemme, 1982) can be used to calculate $Z(T)$ and the effective radius for the comet nucleus becomes $R_{N}=\left[Q\left(H_{2} O\right) /\left(2 \pi Z(T) \cdot f_{A A}\right)\right]^{1 / 2}$.

There are comets where $f_{A A}$ determined following this procedure exceeds 1 without night-side emission, the so-called hyperactive comets ( $A$ 'Hearn et al., 2011; e.g., 103P/Hartley 2, 46P/Wirtanen, and 21P/Gicobini-Zinner). These comets have relatively small nuclei with large amounts of icy grains in their comae that sublimate near the nucleus, providing a large fraction of the total water gas production. Comet Holmes was not a hyperactive comet prior to the outburst. For a radius $R_{N}=2.0 \mathrm{~km}$ obtained from the previous apparition by Tancredi et al. (2000), we find the fraction of active area as $f_{A A}<0.1$ for $2.0<\mathrm{r}(\mathrm{AU})<2.44$ outbound from perihelion in 2007, so the preceding development should provide a reliable estimate of its active surface area and effective radius pre-outburst as shown in Figures 2 and 3. This should generally hold even if the nucleus became slightly smaller during the 2007 apparition. During the outburst, there is a steep rise in $\mathrm{f}_{A A}$, up to a maximum of about 500 . We interpret the rise of $f_{A A}$ as the initial release of the driving gas along with entrained icy dust particles from micron-sized grains up to large icy clumps of surface material. In fact, large fragments that broke off of the nucleus were observed shortly after the megaburst (Stevenson et al., 2010). These icy dust particles sublimate forming a distributed source of water in the coma as observed. This is followed by a steep decline in $f_{A A}$ as the initial gas dissipates and fragmentation of icy clumps finishes. Eventually, $f_{A A}$ slowly decays as the distributed water source from the small grains finishes and dissipates, returning to pre-outburst levels. In addition, fresh volatile-rich material is exposed to the surface insolation at the site of the outburst that sublimates normally from the nucleus. This interpretation is consistent with our Pressurized Obstructed Pore (POP) outburst model discussed in more detail in Section 3.6. 
(insert Figure 2)

(insert Figure 3)

\section{Discussion}

The most important rationale in the explanation of cometary outburst mechanisms is to understand what triggers the process. The outburst needs an internal source of energy that should be consistent with the kinetic energy of the expanding material on the order of $10^{12}-10^{16} \mathrm{~J}$ (Hughes, 1990; Gronkowski and Wesolowski, 2015). We summarize several outburst mechanisms and introduce our new qualitative Pressurized Obstructed Pores (POP) model.

\subsection{Subsurface gas pressure}

A plausible scenario is that Comet Holmes was heated by solar insolation during its perihelion passage and subsurface volatiles (e.g., $\mathrm{CO}, \mathrm{CO}_{2}, \mathrm{CH}_{4}$ ) sublimated as the thermal wave propagated to lower surface layers. The resulting internal gas pressure disrupted the comet surface, which led to a dramatic emission of gas and dust observed as the outburst. It is possible that the nucleus of Holmes has unusually high tensile strength that allows gas pressure to build up in the interior before releasing the energy in a sudden outburst upon surface failure (Stevenson et al., 2014).

To produce a rupture of the icy crust, the build-up of subsurface gas pressure needs to be about $1 \mathrm{kPa}$. This pressure is close to the limits set by the tensile strength of water ice. However, this is not achievable with water vapor in the nucleus. The gas pressure of water can't exceed the pressure at the triple point, i.e., $0.611 \mathrm{kPa}$, or the gas will liquefy or turn into a solid at the low temperatures in the interior. In reality, it is probable that water gas pressures below that of the triple point can initiate an outburst since small cracks or voids can form and propagate at lower pressures, leading to a structural failure of the surface material. Reach et al. (2010) suggested that the nucleus must have a tensile strength between $10-100 \mathrm{kPa}$ in order to have survived the 2007 outburst. The surface layers of a comet refreeze to their initial tensile strength, providing for a repetition of the outburst phenomenon. In addition, supervolatiles can escape from void outlets not covered by regolith/debris. Samarasinha (2001) suggest that the regolith/debris obstruct all of the voids outlets, thereby facilitating internal pressure buildup. In addition, thermal 
stresses might have a role to play in the breakup of the cometary surface and may have contributed to the outburst (Altenhoff et al., 2009).

\subsection{Hydrocarbon clathrates release}

When a comet's heliocentric distance approaches $\leq 2.5 \mathrm{AU}, \mathrm{CO}_{2}$ (sublimation temperature $72 \mathrm{~K}$ ) in addition to $\mathrm{CO}(24 \mathrm{~K})$ and $\mathrm{CH}_{4}(31 \mathrm{~K})$ sublimate rapidly. An appreciable concentration of methane might act as a center of stress in the surface region and led to explosive action (Whitney, 1955; Gronkowski and Wesolowski, 2015). Hydrocarbons like $\mathrm{CH}_{4}$ could be stored in the form of clathrates. If $\mathrm{CH}_{4}$ exists in significant abundance in a comet, it could be a parent of the methyl radical $\left(\mathrm{CH}_{3}\right)$, methylene $\left(\mathrm{CH}_{2}\right)$, methlydyne $(\mathrm{CH})$, and methlydyne ion $\left(\mathrm{CH}^{+}\right)$, all of which have been identified in comets.

However, it is difficult to observe $\mathrm{CH}_{4}$. Methane cannot be detected at ultraviolet (UV) wavelengths because its higher electronic states are predissociated and they do not fluoresce. Also, it cannot be detected at radio wavelengths because it is homopolar and has no allowed rotational transitions. In the visible $(\mathrm{V})$ band, only the $\mathrm{CH}(0,0)$ at $3889 \AA$ and $\mathrm{CH}^{+}(1,0)$ at $3954 \AA$ features have been observed so far.

Mumma et al. (1996) were the first to detect the strong near-infrared (NIR) $v_{3}$ vibrational band at $3.3 \mu \mathrm{m}$ of $\mathrm{CH}_{4}$ in comet $\mathrm{C} / 1996 \mathrm{~B} 2$ (Hyakutake). Kawakita et al. (2014) performed high-dispersion NIR spectroscopic observations of comet $\mathrm{C} / 2010$ (Hill) in the $\mathrm{L}$ band $(3.55 \mu \mathrm{m})$ and detected multiple strong lines from $\mathrm{CH}_{4}$ emission. $\mathrm{CH}_{4}$ is now routinely observed by many groups and its mixing ratio relative to water has been determined to range from $0.3 \%$ to $9 \%$ in comets (Mumma and Charnley, 2011; Bockelée-Morvan, 2011; Kawakita et al., 2014). $\mathrm{CH}_{4}$ was also inferred from the analysis of in situ measurements from the Giotto Ion Mass Spectrometer (IMS) to be about 2\% relative to water in Comet 1P/Halley (Allen et al., 1987; Boice et al., 1990). Methane was found to range from $0.13 \%$ (summer hemisphere) to $0.56 \%$ (winter hemisphere) relative to water pre-perihelion in Comet 67P/ChuryumovGerasimenko by in situ measurements from the Rosetta/Rosina instrument (Le Roy et al., 2015).

\subsection{Amorphous to crystalline ice phase transition}

The phase transition from amorphous water ice $\left(2.3 \mathrm{~g} \mathrm{~cm}^{-3}\right)$ to the crystalline allotrope with a density of $0.94 \mathrm{~g} \mathrm{~cm}^{-3}$, occurs at a temperature near $140 \mathrm{~K}$ and must induce severe strains of the order of $20-30 \%$ which will 
pulverize the ice (e.g. Prialnik and Bar-Nun, 1992, for comet P/Halley crystallization of amorphous ice model; see also Yabushita and Hatta, 1987; and Patashnick et al., 1974). Any volume element that undergoes this irreversible (exothermic) phase transition increases its temperature by about 45 K. Gronkowski (2007) has reviewed the potential sources of energy of cometary outbursts and concluded that the hypothesis concerning the amorphous water ice transformation appears to be the most probable one. The crystallization of amorphous ice into cubic ice releases $9 \times 10^{4} \mathrm{~J} \mathrm{~kg}^{-1}$ of energy (Gronkowski, 2005), so $10^{6}-10^{7} \mathrm{~kg}$ of amorphous ice is needed to supply the required energy of an average outburst. This is less than $0.1 \%$ of the estimated amount of volatiles in the nucleus of Holmes. Sekanina $(2008,2009)$ has argued that this mechanism is responsible for the megaburst of Comet $17 \mathrm{P} / \mathrm{Holmes}$ since it is strongly exothermic (yielding the energy needed for such a megaburst) and is consistent with the range of surface temperatures that are needed for the phase transition at the observed distance and time of the outburst.

If supervolatiles such as $\mathrm{CO}$ and/or $\mathrm{CO}_{2}$ are trapped within the amorphous ice and heated sufficiently, the resulting gas production may be able to drive such activity, if the gas can build up sufficient internal pressure (Stevenson et al., 2014, and references therein).

However, Huebner (2008) supposed that it is quite possible that amorphous ice cannot survive very long on surface and that its existence in comet nuclei still needs to be confirmed. Belton (2010) noted that there is no observational confirmation of the presence of amorphous ice in cometary nuclei and considered its search in the interior of JFCs a major goal for future space missions. Gronkowski and Sacharczuk (2010) suggested that it is questionable that amorphous water ice can survive in the nucleus of $17 \mathrm{P} /$ Holmes because it belongs to the Jupiter-family comets and such comets should convert all of the water ice in the nucleus into a crystalline form after several hundred orbits (Ipatov and A'Hearn, 2011).

\subsection{Polymerization of hydrogen cyanide}

Hydrogen cyanide $(\mathrm{HCN}, 95 \mathrm{~K})$, a well-known minor constituent of interstellar ice grain mantles, was marginally detected by Huebner et al. (1974) in comet C/1973 E1 (Kohoutek) at $3.3 \mathrm{~mm}(88.63 \mathrm{GHz}$ ) wavelength (J=1-0 ground state rotational transition) and appears to be the prime parent of $\mathrm{CN}$ $\left(\mathrm{HCN}+\mathrm{hv} \rightarrow \mathrm{CN}+\mathrm{H}+\mathrm{E}_{\mathrm{k}} ; \mathrm{HCN}+\mathrm{e}^{-} \rightarrow \mathrm{CN}+\mathrm{H}+\mathrm{e}^{-}\right)$at least in some comets 
(Huebner et al., 1989), including 1P/Halley (Schloerb et al., 1987; BockeléeMorvan et al., 1987; Winnberg et al., 1987), 17P/Holmes (Bockelée-Morvan et al., 2008), C/2013 R1 (Lovejoy) (Opitom et al., 2015), and many other examples. Since polymerization is an important exothermic process that links simple organics to prebiotic molecules, Rettig et. al. (1992) investigated the possibility that HCN polymerization can provide the energy needed for outbursts. They concluded that HCN polymerization in an inhomogeneous cometary nucleus of is a possible source of energy for outbursts.

\subsection{Cometary Outburst Model by Avalanche (COMA)}

There is a concern in the above mechanisms concerning the randomness of comet Holmes' outbursts: twice in 1892-1893 (separated by about 71 days) and then nothing for 114 years. If the cause of the onset of sudden activity was mainly caused by the heat flux into the interior of the nucleus, then one might expect some, at least minor, increase in the activity on a nearly periodic basis. Therefore, we suggest another possible outburst mechanism, the Cometary Outburst Model by Avalanche (COMA), with the trigger being a large landslide or avalanche. Using images from the Deep Space 1 encounter with Comet 19P/Borrelly, Boice et al. (2002) and Britt et al. (2004) suggested that steep slope failure and mass wasting on mesa terrain exposed volatile-rich material leading to sublimation activity. Thomas et al. (2007) also noted erosion and associated slope retreat as important processes on Comet Tempel 1.

Comet nuclei are not smooth, spherical objects (see, e.g., Comet 67P/Churyumov-Gerasimenko, also a JFC). Some comet nuclei are odd-shaped with unusual surface structures. If a nucleus has a nearly vertical surface structure relative to its center of mass, then it can have a dust cover on this vertical structure that does not collapse in the very low gravity field. However, the slightest disturbance could create a "slow dust avalanche" that exposes the subsurface ice. The "falling" dust can easily be entrained by the sudden onset of gas production from the exposed, vertical surface because the gas does not need to overcome the tensile strength of the surface layer. The disturbance could be caused by the impact of a very small, possible $\mathrm{cm}$-sized, meteoroid, or from a similar-sized particle emitted from the nucleus in a quasi-stable orbit that falls back to the surface. Such impacts are very random. Rosetta images 
have shown movement of surface features that resemble landslides (Groussin et al., 2015), with a probable increase in the measured gas production (Capaccioni, 2015), supporting the COMA mechanism for cometary activity and outbursts. COMA may also account for the activity associated with the comet's steeply sided active "pits" (Vincent et al., 2015).

\subsection{Recrystallization of ice in the surface regolith - the Pressurized Obstructed Pores (POP) model}

There are many possible mechanisms for cometary outbursts, some of which are mentioned above. Here we briefly describe one possible mechanism. We propose the following qualitative model for cometary outbursts that draws from several ideas summarized in the preceding discussion. We do not attempt to model all of the data at this time, rather, we merely explore whether the mechanism is a plausible one for explaining some cometary outbursts, especially at large heliocentric distances. We believe the key feature of our model missing from previous ones that suppose the release of subsurface volatiles (e.g., Whipple, 1963; Samarasinha, 2001; Prialnik et al., 2008; Kossacki and Szutowicz, 2011) is the recrystalization of water near the surface that plugs the pores and prevents gas from escaping the nucleus, allowing interior gas pressure to increase and eventually triggering the outburst. Of course, we can't preclude other mechanisms, especially for minor outbursts or disruptions that occur pre-perihelion (e.g., comets D/1999 S4 (LINEAR), C/2012 S1 (ISON), 73P/Schwassmann-Wachmann 3).

We know that cometary material is very porous leading to very low thermal conductivity (and high thermal inertia) and that the surface is covered by a thin dusty regolith (centimeters to a few meters) with very limited amount of surface water ice (see, e.g., Prialnik et al., 2004; Weissman et al. 2004, for reviews). Since the thermal skin depth due to diurnal variations (Keller, 1990) is about $4.4 \mathrm{~cm}$ for comet Holmes, we can expect water depletion in the dusty regolith to about this depth. The basis of our model is the asymmetric thermal environment of the interior of the nucleus, pre- and post-perihelion. The solar energy input is symmetric about perihelion but the nature of the temperature gradient into the porous interior differs due to thermal inertia and energy flow. As the comet approaches the Sun, the average surface temperature increases 
as heat flows into the nucleus, warming the subsurface layers, and releasing volatiles. The interior cools by gas flow at sublimation fronts and (primarily) conductive heat flow within the matrix. The maximum energy impacting the surface occurs at perihelion with a steep temperature gradient into the interior. On the outbound journey, the surface cools due to the decreasing solar irradiation while the interior temperature remains relatively unchanged due to the long heat diffusion time (high thermal inertia) so volatiles (and supervolatiles) continue to be released. As the comet recedes further from the Sun, the temperature gradient becomes less steep as the surface temperature drops and the heat flow from the interior to the surface (primarily via gas flow) decreases. Far from the Sun, the temperature gradient may even invert as the surface and interior cools and gas flow is negligible, allowing the residual internal heat to escape and cooling the nucleus around aphelion. All of this occurs within the upper tens of meters of the surface layers since the orbital skin depth is typically about $18 \mathrm{~m}$ for a JFC (Prialnik et al., 2004).

Within the heliocentric distance range of 2-3 $\mathrm{AU}$, the situation is compounded by the gas to solid sublimation of water in the near-surface regolith, potentially closing pores and blocking the release of volatiles from the interior. After enough of the regolith pores are plugged, pressure builds in the interior until it reaches sufficient levels to blow off the surface layers, resulting in an outburst. If the pressure build-up is great enough, this may lead to the total destruction of the comet. Since the pressure build-up depends on a sufficient number of pores being plugged in a specific region to create a "seal" that blocks the escaping gas from subsurface layers, it is a stochastic mechanism due to the indeterminacy in which the pore-closing process may evolve and may not repeat periodically at every apparition. This process can occur at distances closer to the Sun in shaded regions where the surface temperature is already sufficiently cool to allow the recondensation of water. The presence of water ice in the surface layers of 67P/ChuryumovGerasimenko has been suggested by MIRO observations onboard Rosetta (Choukroun et al., 2015) of the polar night in the southern regions of the nucleus. It can also occur on a diurnal cycle since this scenario is repeated on a smaller time scale as the surface goes through the evening terminator to the night side of the nucleus. Recently, De Sanctis et al. (2015) observed a diurnal 
cycle of water ice on the surface of comet 67P/Churyumov-Gerasimenko, confirming this long-held notion. The recrystallization of water blocking pores in the surface regolith could lead to mini-outbursts that have been observed on the night side of comets 19P/Borrelly (Boice et al., 2002; Soderblom et al., 2004) and possibly those seen at sunrise on 9P/Tempel 1 ( $A^{\prime}$ Hearn et al., 2005; Prialnik et al., 2008), although Lisse et al. (2013) summarize that none occurred near dawn and had a preference to occur when the surface was cooling. The POP model predicts that outbursts should occur with more frequency postperihelion as observed (Hughes, 1991) and that outbursts should occur on the night side, just past the evening terminator with more regularity, consistent with Lisse et al. (2013). During an outburst, the material that is blown off should consist of clumpy grains held together by water ice that would sublimate and fragment in the coma, leading to a distributed source of water. In addition, fresh surface area is exposed so volatiles and supervolatiles can sublimate normally from the nucleus at the site of the outburst. It is also consistent with the pre- and post-perihelion asymmetries in the "Christmas tree" behavior of the production rates of volatiles seen by Biver et al. (1997) in comet C/1995 01 (Hale-Bopp). We would expect that the production rates of volatiles would remain higher on the outbound portion of the orbit, relative to inbound, since the interior is warmer in post-perihelion due to the thermal inertia of the nucleus.

\section{Concluding Remarks}

According to our present knowledge of the physical and chemical structure of comets, the driver of cometary activity is the production of gas-phase molecules - mainly $\mathrm{H}_{2} \mathrm{O}$, which lifts the dust particles from the nucleus, so a correlation between $\mathrm{H}_{2} \mathrm{O}$ and dust production is expected.

Our derived water production rate $\left(14,960 \mathrm{~kg} \mathrm{~s}^{-1}\right)$, for comet 17P/Holmes, based on visual magnitude measurements using the SEMVM model (see Table 1 and Figure 1), is in very good agreement with Schleicher (2009), who derived an average water production rate of $5 \times 10^{29}$ molecules $\mathrm{s}^{-1}$, based on $\mathrm{OH}$ measurements (see Eq. 3) at nearly same time after the megaburst.

The occurrence of an outburst represents a dramatic departure from thermal equilibrium between the comet and space. It is considered that outbursts are mainly caused by internal processes occurring in porous comet 
Acknowledgements. This work was supported by FAPESP, São Paulo, Brazil, under Grant No. 2015/03176-8 and National Science Foundation Planetary Astronomy Program Grant No. 0908529. The authors sincerely thank two anonymous reviewers for their very constructive comments and suggestions that have helped to improve the quality of the earlier version of this manuscript.

\section{References}

A'Hearn, M. F., Millis, R. L., Schleicher, D. G., et al., 1995. The ensemble properties of comets: results from narrowband photometry of 85 comets. Icarus, 118, 223-270.

A'Hearn, M.F., Belton, M.J.S., Delamere, W.A., et al., 2005. Deep Impact: excavating Comet Tempel 1. Science, 310, 258-264. 
A'Hearn, M.F., Belton, M.J.S., Delamere, W.A., et al., 2011. EPOXI at Comet Hartley 2. Science, 332, 1396-1400.

Allen, M., Delitsky, M., Huntress, W., et al., 1987. Evidence for methane and ammonia in the coma of comet P/Halley. Astron. Astrophys., 187, 502-512.

Altenhoff, W.J., Kreysa, E., Menten, K.M., et al., 2009. Why did Comet 17P/Holmes burst out? Astron. Astrophys., 495, 975-978.

Belton, M.J.S., 2010. Cometary activity, active areas, and a mechanism for collimated outflows on 1P, 9P, 19P, and 81P. Icarus, 210, 881-897.

Biver, N., Bockelée-Morvan, D., Colom, P., et al., 1997. Long-term evolution of the outgassing of Comet Hale-Bopp from radio observations. Earth, Moon and Planets, 78, 5-11.

Bockelée-Morvan, D., Crovisier, J., Despois, D., et al., 1987. Molecular observations of comets P/Giacobini-Zinner 1984e and P/Halley 1982i at millimetre wavelengths. Astron. Astrophys., 180, 253-262.

Bockelée-Morvan, D., Biver, N., Jehin, E., et al., 2008, Large excess of heavy nitrogen in both hydrogen cyanide and cyanogen from Comet 17P/Holmes. Astrophys. J., 679, L49-L52.

Bockelée-Morvan, D., 2011. An Overview of Comet Composition. In The Molecular Universe, Proceedings IAU Symposium No. 280, (J. Cernicharo and R. Bachiller, Eds.), pp. 261-274.

Boice, D.C., Huebner, W.F., Sablik, M.J., Konno, I., 1990. Distributed coma sources and the $\mathrm{CH}_{4} / \mathrm{CO}$ ratio in Comet Halley. Geophys. Res. Lett., 17 (11), 1813-1816.

Boice, D.C., Soderblom, L.A., Britt, D.T., et al., 2002. The Deep Space 1 encounter with Comet 19P/Borrelly. Earth, Moon and Planets, 89, 301-324.

Britt, D.T., Boice, D.C., Buratti, B.J. et al., 2004. The morphology and surface processes of Comet 19P/Borrelly. Icarus, 167, 45-53.

Capaccioni, F., 2015, 2nd COSPAR Symposium: Water and Life in the Universe, Session 4: Water and Life in the Solar System, 9-13 November, Foz do Iguaçu, Brazil. 
Choukroun, M., Keihm, S., Schloerb, F.P., et al., 2015. Dark side of comet 67P/Churyumov-Gerasimenko in Aug.-Oct. 2014. Astron. Astrophys., 583, A28.

Cowan, J.J., A'Hearn, M.F., 1979. Vaporization of comet nuclei: light curves and life times. Moon Planets, 21, 155-171.

de Almeida, A.A., Singh, P.D., Huebner, W.F., 1997. Water release rates, active areas, and minimum nuclear radius derived from visual magnitudes of comets an application to comet 46P/Wirtanen. Planet. Space Sci., 45, 681-692.

de Almeida, A.A., Sanzovo, G.C., Singh, P.D., et al., 2007. On the relationship between visual magnitudes and gas and dust production rates in target comets to space missions. Adv. Space Res., 39, 432-445.

Delsemme, A.H., 1982. Chemical composition of cometary nuclei. In Comets, (Wilkening, L. L., Ed.), University of Arizona Press, Tucson, pp. 85-130.

De Sanctis, M.S., Capaccioni, F., Ciarniello, M., et al., 2015. The diurnal cycle of water ice on comet 67P/Churyumov-Gerasimenko. Nature, 525, 500-503.

Divine, N., 1981. Numerical models for Halley dust environments. In The comet Halley dust and gas environments, (Battrick, B. and Swallow, E., Eds.), ESTEC, Noordwijk, The Netherlands, ESA SP-174, 47, pp. 25-30.

Gronkowski, P., 2005. The source of energy of the comet 29P/SchwassmannWachmann 1 outburst activity: the test of the summary. Mon. Not. R. Astron. Soc., 360, 1153-1161.

Gronkowski, P., 2007. The search for a cometary outbursts mechanism: a comparison of various theories. Astron. Nachr., 328, 126-136.

Gronkowski, P., Sacharczuk, Z., 2010. Cometary outbursts - a search for a cause of the comet 17P/Holmes outburst. Mon. Not. R. Astron. Soc., 408, 1207-1215.

Gronkowski, P., Wesolowski, M., 2015. A model of cometary outbursts: a new simple approach to the classical question. Mon. Not. R. Astron. Soc., 451, 30683077.

Groussin, O., Sierks, H., Barbieri, C., et al., 2015. Temporal morphological changes in the Imhotep region of comet 67P/Churyumov-Gerasimenko. Astron. Astrophys., 583, A36-. 
Huebner, W.F., Snyder, L.E., Buhl, D., 1974. HCN radio emissions from Comet Kohoutek (1973f). Icarus, 23, 580-584.

Huebner, W.F., Boice, D.C., Korth, A., 1989. Halley's polymeric organic molecules. Adv. Space Res., 9 (2), 29-34.

Huebner, W.F., Keady, J.J., Lyon, S.P., 1992. Solar photo rates for planetary atmospheres and atmospheric pollutants. Astrophys. Space Sci., 195, 1-294.

Huebner, W. F., 2008. Origins of cometary materials. Space Sci. Rev., 138, 5-25.

Hughes, D.W., 1990. Cometary outbursts: a review. Q. Jl. astr. Soc., 31, 69-94.

Hughes, D.W., 1991. Possible mechanisms for cometary outbursts. In Comets in the Post-Halley Era, Vol. 2, (Newburn, R.L. Jr., Neugebauer, M., Rahe, J., Eds.), Kluwer, Dordrecht, pp. 825-851.

International Comet Quarterly Archive of Photometric Data on Comets (Smithsonian Astrophysical Observatory).

Ipatov, S.I., A'Hearn, F., 2011. The outburst triggered by the Deep Impact collision with Comet Tempel 1. Mon. Not. R. Astron. Soc., 414, 76-107.

Kawakita, H., Dello Russo, N., Vervack, R. Jr., et al., 2014. Extremely organic-rich coma of Comet C/2010 G2 (Hill) during its outburst in 2012. Astrophys. J., 788, 110 (7pp).

Keller, H.U., 1990, The Nucleus. In Physics and Chemistry of Comets. (W.F. Huebner, Ed.), Springer-Verlag, Berlin, New York, Tokyo, pp. 13-68.

Kossacki, K.J., Szutowicz, S., 2011. Comet 17P/Holmes: possibility of a CO driven explosion. Icarus, 212, 847-857.

Le Roy, L., Altwegg, K., Balsiger, H., et al., 2015. Inventory of the volatiles on comet 67P/Churyumov-Gerasimenko from Rosetta/ROSINA. Astron.

Astrophys., 583, A1.

Lisse, C., Bar-Nun, A., Laufer, D., et al., 2013. Cometary ices. In The Science of Solar System Ices, (Gudipati, M.S. and Castillo-Rogez, J., Eds.), Springer, New York, pp. 455-485.

Morris, C. S., 1973. On aperture corrections for comet magnitude estimates. PASP, 85, 470-473. 
Mumma, M.J., DiSanti, M.A., Dello Russo, N., et al., 1996. Detection of abundant ethane and methane, along with carbon monoxide and water, in Comet C/1996 B2 Hyakutake: evidence for interstellar origin. Science, 272, 1310-1314.

Mumma, M.J., Charnley, S.B., 2011. The chemical composition of comets emerging taxonomies and natal heritage. Ann. Rev. Astron. Astrophys., 49, 471524.

Newburn Jr., R. L., 1981. A semi-empirical photometric theory of cometary gas and dust production: application to Comet Halley's gas production rates. In The Comet Halley Dust and Gas Environment, ESTEC, Noordwijk, The Netherlands, ESA SP-174, pp. 3-18.

Ney, E. P., 1982. Optical and infrared observations of bright comets in the range $0.5 \mu \mathrm{m}$ to $20 \mu \mathrm{m}$. In Comets, (Wilkening, L. L., Ed.), University of Arizona Press, Tucson, pp. 323-340.

Opitom, C., Jehin, E., Manfroid, J., et al., 2015. TRAPPIST photometry and imaging monitoring of comet C/2013 R1 (Lovejoy): implications for the origin of daughter species. Astron. Astrophys. 584, A121.

Patashnick, M., Rupprecht, G., Schuerman, D. W., 1974. Energy source for comet outbursts. Nature, 250, 313-314.

Prialnik, D., Bar-Nun, A., 1992. Crystallization of amorphous ice as the cause of comet Halley's outburst at 14 AU. Astron. Astrophys., 258, L9-L12.

Prialnik, D., Benkhoff, J., Podolak, M., 2004. Modeling the structure and activity of cometary nuclei. In Comets II, (Festou, M.C., Keller, H.U., and Weaver, H.A., Eds.), University of Arizona Press, Tucson, pp. 359-387.

Prialnik, D., A'Hearn, M.F., Meech, K.J., 2008. A mechanism for short-lived cometary outbursts at sunrise as observed by Deep Impact on 9P/Tempel 1. Mon. Not. R. Astron. Soc., 388, L20-L23.

Randall, C.E., Schleicher, D.G., Ballou, R.G., Osip, D.J., 1992. Observational constraints on molecular scale lengths and lifetimes in comets. BAAS, 24, 1002.

Reach, W.T., Vaubaillon, J., Lisse, C.M., et al., 2010. Explosion of Comet 17P/Holmes as revealed by the Spitzer Space Telescope. Icarus, 208, 276-292.

Rettig, T.W., Tegler, S.C., Pasto, D.J., Mumma, M.J., 1992. Comet outbursts and polymers of HCN. Astrophys. J., 398, 293-298. 
Rousselot, P., Hill, S.M., Burger, M.H., et al., 2000. Theoretical modeling of the $\mathrm{C}_{2}$ fluorescence spectrum in Comet Hale-Bopp. Icarus, 146, 263-269.

Samarasinha, N.H., 2001. A model for the breakup of Comet LINEAR (C/1999 S4). Icarus, 154, 540-544.

Sanzovo, G.C., Singh, P.D., Huebner, W.F., 1996. Dust colors, dust release rates, and dust-to-gas ratios in the comae of six comets. Astron. Astrophys. Suppl. Ser., 120 (2), 301-311.

Sanzovo, G. C., de Almeida, A. A., Misra, A., et al., 2001. Mass-loss rates, dust particle sizes, nuclear active areas and minimum nuclear radii of target comets for missions STARDUST and CONTOUR. Mon. Not. R. Astron. Soc., 326, 852-868.

Sanzovo, G.C., Trevisan Sanzovo, D., de Almeida, A.A., 2010. On the relationship between gas and dust in 15 comets: an application to Comet 103P/Hartley 2 target of the NASA EPOXI mission of opportunity. In Icy Bodies of the Solar System (Fernández, J.A., Lazzaro, D., Prialnik, D., and Schulz, R., Eds.), Proceedings of IAU Symposium, No. 263, pp. 272-276.

Schleicher, D. G., 2009. The long-term decay in production rates following the extreme outburst of comet 17P/Holmes. Astron. J., 138, 1062-1071.

Schloerb, F.P., Kinzel, W.M., Swade, D.A., Irvine, W.M., 1987. Observations of $\mathrm{HCN}$ in comet P/Halley. Astron. Astrophys., 187, 475-480.

Sekanina, Z., 2008. Exploding Comet 17P/Holmes. International Comet Quarterly, 30, 3-28.

Sekanina, Z., 2009. Comet 17P/Holmes: a megaburst survivor. International Comet Quarterly, 31, 5-23.

Singh, P.D., Huebner, W.F., Costa, R.D.D., et al., 1997. Gas and dust release rates and color of dust in comets P/Halley (1986 III), P/Giacobini-Zinner (1985 XIII), and P/Hartley-Good (1985 XVII). Planet. Space Sci., 45 (4), 455-467.

Soderblom, L.A., Boice, D.C., Britt, D.T., et al., 2004. Imaging Borrelly. Icarus, 167, 4-15.

Stevenson, R., Kleyna, J., Jewitt, D., 2010. Transient fragments in outbursting comet 17P/Holmes. Astron. J., 139, 2230-2240.

Stevenson, R., Bauer, J.M., Kramer, E.A., et al., 2014. Lingering grains of truth around Comet 17P/Holmes. Astrophys. J., 787:116 (6pp). 
Tancredi, G., Fernández, J.A., Rickman, H., Licandro, J., 2000. A catalog of observed nuclear magnitudes of Jupiter family comets. Astron. Astrophys. Suppl. Ser., 146, 73-90.

Thomas, P.C., Veverka, J., Belton, M.J.S., et al., 2007. The shape, topography, and geology of Tempel 1 from Deep Impact observations. Icarus, 187, 4-15.

Vincent, J.-B., Bodewits, D., Besse, S., et al., 2015. Large heterogeneities in comet $67 \mathrm{P}$ as revealed by active pits from sinkhole collapse. Nature, 523, 6366.

Weissman, P.R., Asphaug, E., Lowry, S.C., 2004. Structure and density of cometary nuclei. In Comets II, (Festou, M.C., Keller, H.U., and Weaver, H.A., Eds.), University of Arizona Press, Tucson, pp. 337-357.

Whipple, F., 1963 On the structure of the cometary nucleus. In The Moon, Meteorites, and Comets, Solar System Vol. 4 (Middlehurst, B. and Kuiper, G.P. Eds.), Chicago: The University of Chicago Press, Chicago, p. 639-664.

Whitney, C., 1955. Comet outbursts. Astrophys. J., 122, 190-195.

Winnberg, A., Ekelund, L., Ekelund, A., 1987. Detection of HCN in comet P/Halley. Astron. Astrophys., 172, 335-341.

Yabushita, S., Hatta, N., 1987. A thermal modelling of cometary activity with a crystallized water ice nucleus. Earth, Moon and Planets, 37, 141-146.

\section{Captions:}

Table 1. Compared observing circumstances and derived water production rates for Comet 17P/Holmes.

Table 2. Total gas and dust estimates for Comet 17P/Holmes outburst ejecta.

Figure 1. Water production rates calculated by the SEMVM model for 17P/Holmes before and after the megaburst that occurred during 23 and 24 October 2007, about 173 days after perihelion. The comet achieved an average water production rate of $5 \times 10^{29}$ molecules $s^{-1}$ during the megaburst, in very good agreement with Schleicher (2009). 
Figure 2. The fraction of active surface area $\left(f_{A A}\right)$ inferred using the SEMVM model as a function of heliocentric distance for comet 17P/Holmes. Note the small active fraction $(<0.1)$ pre-outburst and the large increase of $\mathrm{f}_{\mathrm{AA}}$ to about 500 after the megaburst at $2.44 \mathrm{AU}$.

Figure 3. Enlarged area of Figure 2 around the megaburst at $2.44 \mathrm{AU}$ to show details in the changes of $f_{A A}$. Note the rapid increase in $f_{A A}$ during the outburst, followed by the steep decline and then gradual decay after about $2.5 \mathrm{AU}$. 
Table 1. Compared Observing Circumstances and Derived Water Production Rates for Comet 17P/Holmes.

\begin{tabular}{|c|c|c|c|c|c|c|c|c|c|c|c|}
\hline $\begin{array}{c}\text { UT Date } \\
\text { (Schleicher,2009) }\end{array}$ & $\begin{array}{l}\Delta T^{a} \\
\text { (day) }\end{array}$ & $\begin{array}{c}r_{H} \\
(\mathrm{AU})\end{array}$ & $\begin{array}{l}\text { Phase } \\
\text { Angle }\left(^{\circ}\right)\end{array}$ & $\begin{array}{c}\mathrm{v} \\
\left(\mathrm{km} \cdot \mathrm{s}^{-1}\right)\end{array}$ & $\begin{array}{l}\mathrm{Q}\left(\mathrm{H}_{2} \mathrm{O}\right) \\
\left(\mathrm{mol} . \mathrm{s}^{-1}\right)\end{array}$ & $\begin{array}{c}\text { UT Date } \\
\text { (This work) }\end{array}$ & $\begin{array}{r}\Delta T^{a} \\
\text { (day) }\end{array}$ & $\begin{array}{l}r_{H} \\
(\mathrm{AU})\end{array}$ & $\begin{array}{c}\text { Phase } \\
\text { Angle }\left({ }^{\circ}\right)\end{array}$ & $\begin{array}{c}\mathrm{v} \\
\left(\mathrm{km} \cdot \mathrm{s}^{-1}\right)\end{array}$ & $\begin{array}{r}\mathrm{Q}\left(\mathrm{H}_{2} \mathrm{O}\right) \\
\left(\mathrm{mol} . \mathrm{s}^{-1}\right)\end{array}$ \\
\hline 7 Nov 1.20 & +180.67 & 2.468 & 15.0 & +6.7 & $3.467(29)$ & 2007 Nov 1.21 & +180.71 & 2.4675 & 14.9 & +6.73 & $1.814(29)$ \\
\hline 2007 Nov 11.19 & +190.66 & 2.507 & 12.7 & +6.9 & $1.096(29)$ & 2007 Nov 11.21 & +190.71 & 2.5068 & 12.7 & +6.88 & $6.491(28)$ \\
\hline 2007 Nov 20.19 & +199.66 & 2.543 & 11.5 & +7.0 & $5.495(28)$ & 2007 Nov 20.21 & +199.71 & 2.5429 & 11.4 & +7.00 & $4.210(28)$ \\
\hline 2007 Dec 3.36 & +212.83 & 2.597 & 11.5 & +7.1 & $2.455(28)$ & 2007 Dec 3.27 & +212.77 & 2.5963 & 11.5 & +7.15 & $1.820(28)$ \\
\hline 2007 Dec 4.28 & +213.75 & 2.601 & 11.6 & +7.2 & $2.138(28)$ & 2007 Dec 4.28 & +213.78 & 2.6005 & 11.6 & +7.16 & $2.027(28)$ \\
\hline 2007 Dec 5.27 & +214.74 & 2.605 & 11.7 & +7.2 & $1.995(28)$ & 2007 Dec 5.17 & +214.67 & 2.6042 & 11.7 & +7.17 & $1.950(28)$ \\
\hline 2008 Jan 1.23 & +241.70 & 2.718 & 11.7 & +7.4 & $7.762(27)$ & 2008 Jan 1.19 & +241.69 & 2.7178 & 16.2 & +7.37 & $4.510(27)$ \\
\hline 2008 Mar 4.21 & +304.68 & 2.990 & 19.1 & +7.5 & $1.862(27)$ & 2008 Mar 4.76 & +305.26 & 2.9922 & 19.1 & +7.50 & $7.701(25)$ \\
\hline 2008 Mar 5.16 & +305.63 & 2.994 & 19.1 & +7.5 & $8.511(26)$ & 2008 Mar 5.00 & +305.50 & 2.9932 & 19.1 & +7.50 & $1.085(26)$ \\
\hline
\end{tabular}

${ }^{\mathrm{a}}$ Time from perihelion, 2007 May 4.50; the onset of the outburst occurred at about 2007 October 23.7 , or $\Delta T=+172.2 ; 3.467(29)=3.467 \mathrm{x} 10^{29}$. 
Table 2. Total gas and dust estimates for comet 17P/Holmes outburst ejecta.

\begin{tabular}{ccccc}
\hline $\begin{array}{c}\text { UT Date } \\
\text { (This work) }\end{array}$ & $\begin{array}{c}\mathrm{Q}_{\mathrm{g}}{ }^{\mathrm{a}} \\
\left(\mathrm{kg} \cdot \mathrm{s}^{-1}\right)\end{array}$ & $\begin{array}{c}\mathrm{Q}_{\mathrm{d}}{ }^{\mathrm{b}} \\
\left(\mathrm{kg} \cdot \mathrm{s}^{-1}\right)\end{array}$ & $\begin{array}{c}\mathrm{Q}_{\mathrm{t}}{ }^{\mathrm{c}} \\
\left(\mathrm{kg} \cdot \mathrm{s}^{-1}\right)\end{array}$ & $\chi^{\mathrm{d}}$ \\
\hline 2007 Nov 1.21 & $6.186 \times 10^{3}$ & $5.505 \times 10^{3}$ & $1.169 \times 10^{4}$ & 0.890 \\
2007 Nov 11.21 & $2.213 \times 10^{3}$ & $2.101 \times 10^{3}$ & $4.314 \times 10^{3}$ & 0.949 \\
2007 Nov 20.21 & $1.436 \times 10^{3}$ & $1.401 \times 10^{3}$ & $2.837 \times 10^{3}$ & 0.976 \\
2007 Dec 3.27 & $6.206 \times 10^{2}$ & $6.381 \times 10^{2}$ & $1.259 \times 10^{3}$ & 1.028 \\
2007 Dec 4.28 & $6.912 \times 10^{2}$ & $7.059 \times 10^{2}$ & $1.397 \times 10^{3}$ & 1.021 \\
2007 Dec 5.17 & $6.650 \times 10^{2}$ & $6.808 \times 10^{2}$ & $1.346 \times 10^{3}$ & 1.024 \\
2007 Jan 1.19 & $1.538 \times 10^{2}$ & $1.726 \times 10^{2}$ & $3.264 \times 10^{2}$ & 1.122 \\
2008 Mar 4.76 & $2.626 \times 10^{0}$ & $3.805 \times 10^{0}$ & $6.431 \times 10^{0}$ & 1.449 \\
2008 Mar 5.00 & $3.700 \times 10^{0}$ & $5.248 \times 10^{0}$ & $8.948 \times 10^{0}$ & 1.418 \\
\hline
\end{tabular}

${ }^{a}$ Gas mass release rates obtained from Table 1 using Equation (4) of this work.

${ }^{\mathrm{b}}$ Dust mass release rates derived from the dust-to-gas correlation by Sanzovo et al., 2009.

${ }^{c}$ Total mass of materials ejected during the 2007 outburst.

${ }^{\mathrm{d}}$ Dust-to-gas mass ratio. 


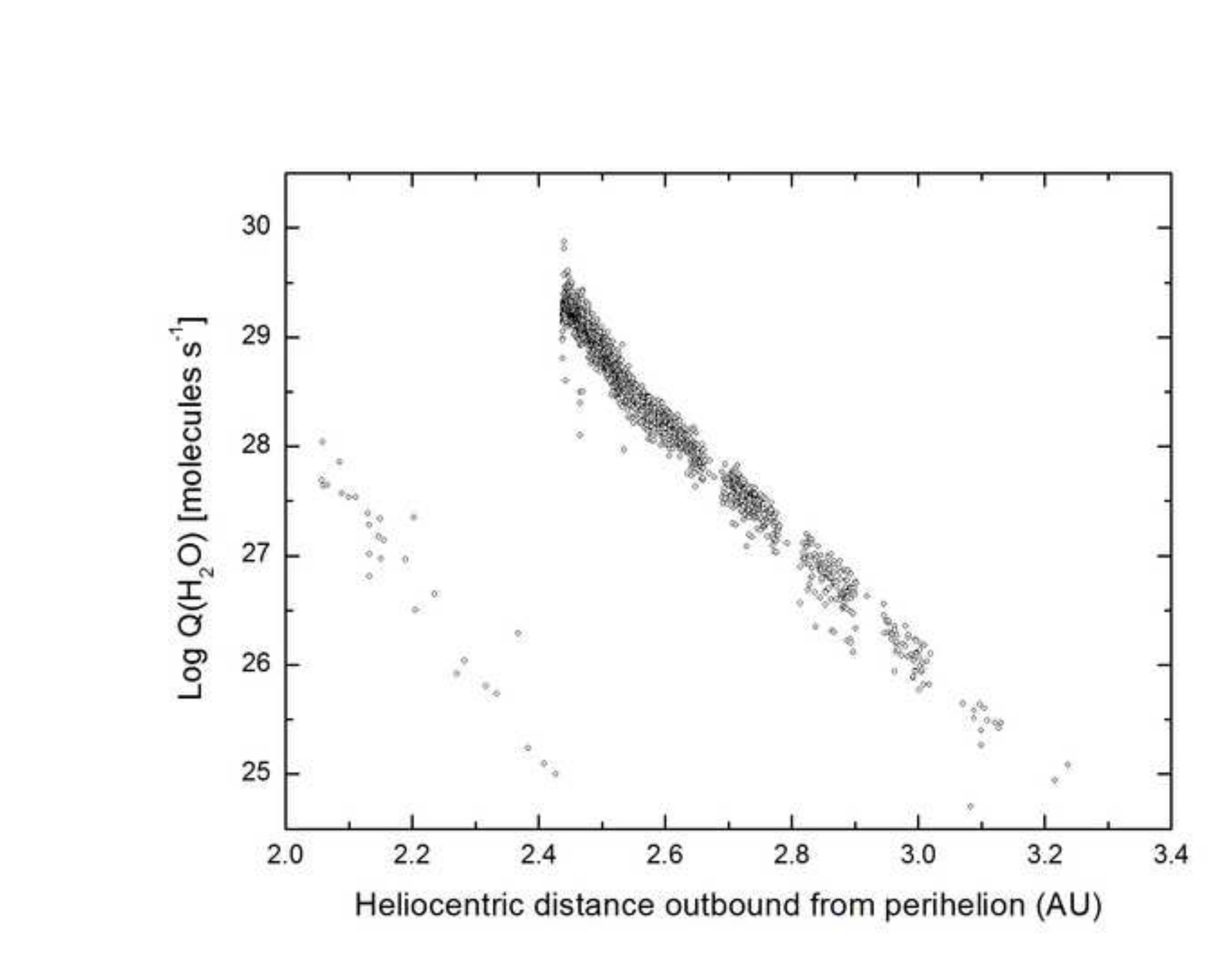

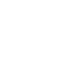

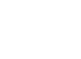

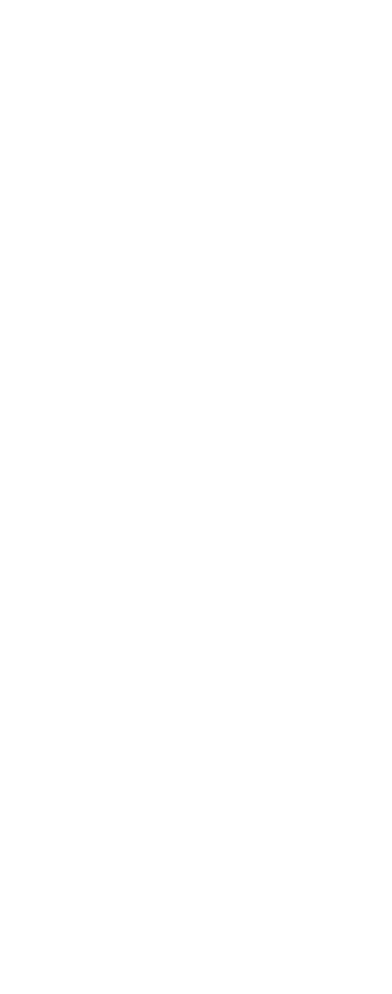

(1)

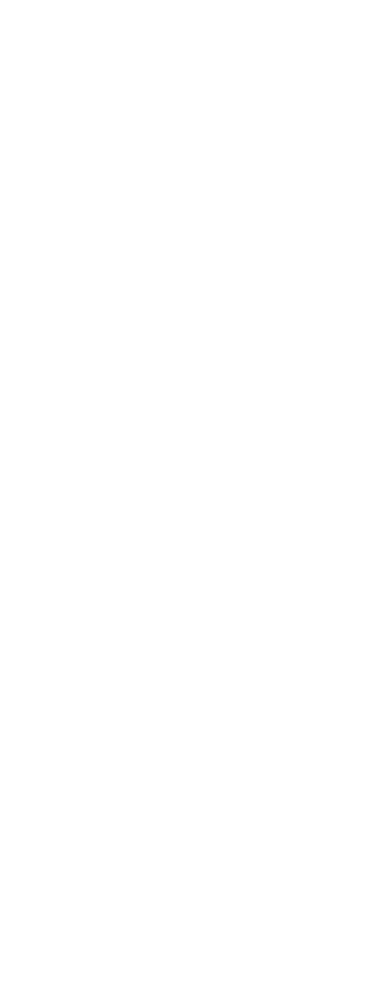

.

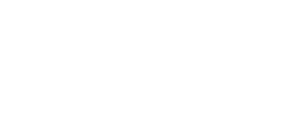

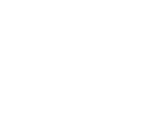

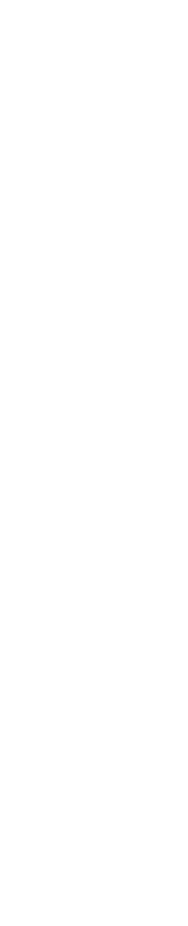

(1)

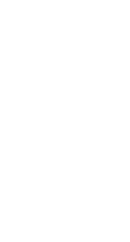
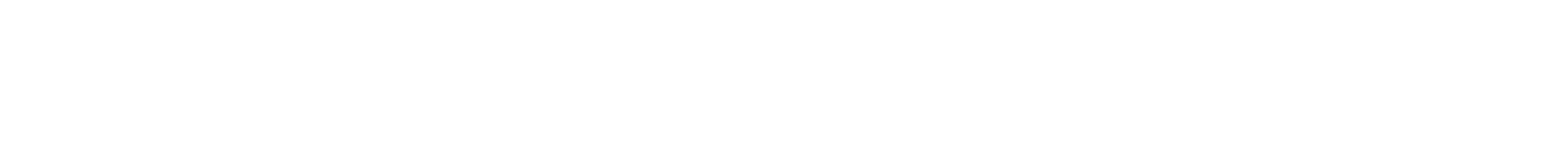


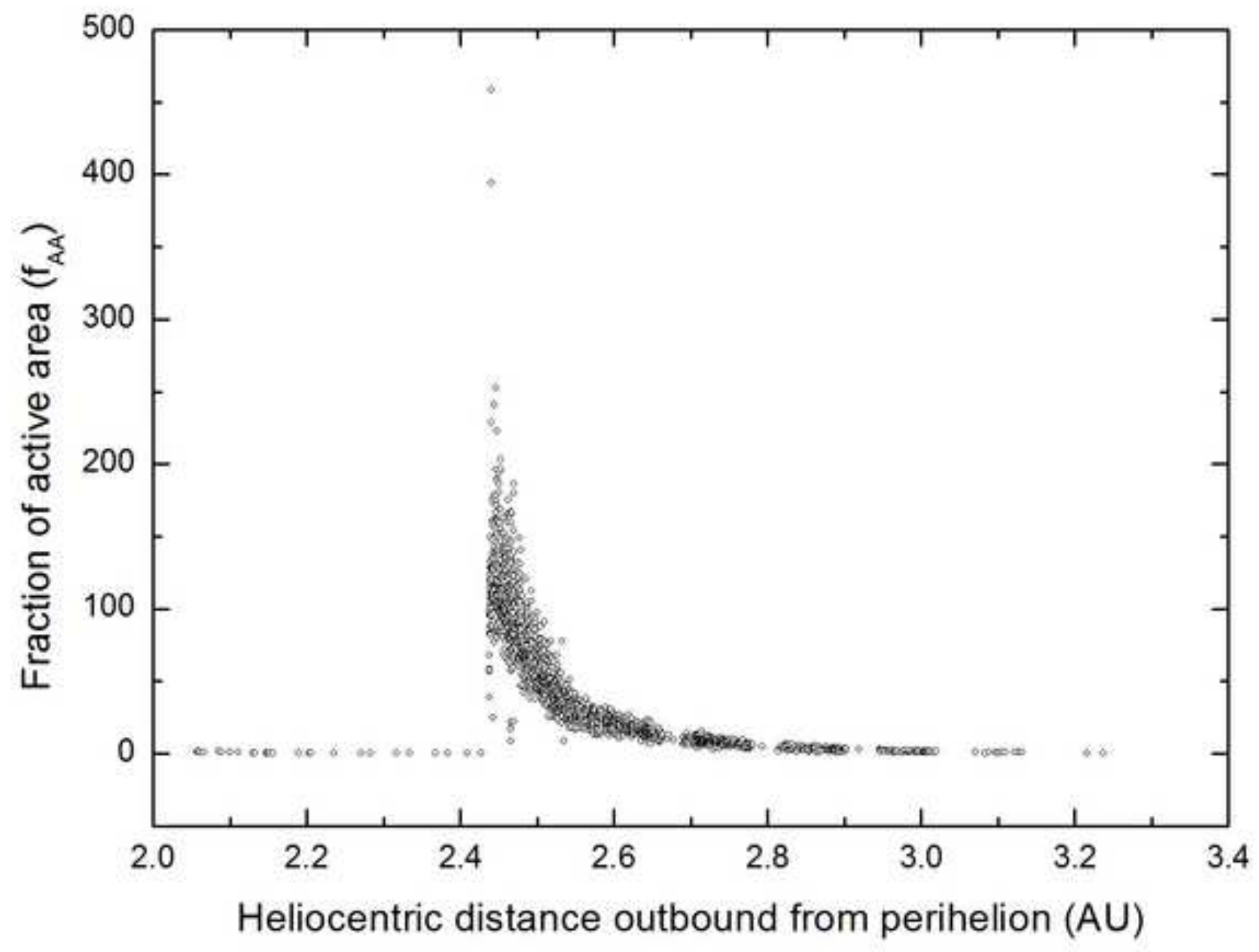




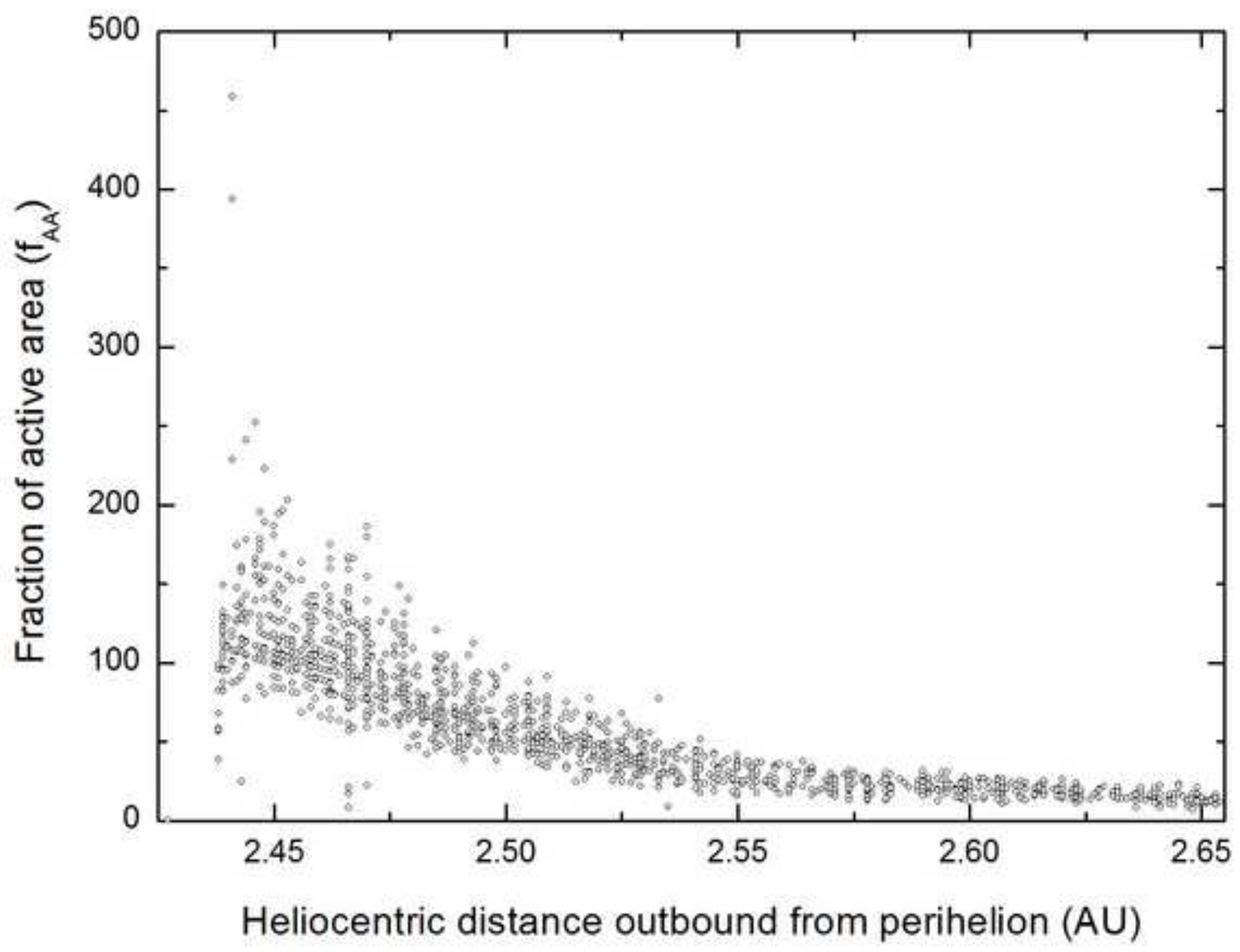

